\title{
Spin liquid versus long-range magnetic order in the frustrated body-centered-tetragonal lattice
}

\author{
Carlene Farias, ${ }^{1,2,3,4}$ Christopher Thomas, ${ }^{5}$ Catherine Pépin, ${ }^{6}$ Alvaro Ferraz, ${ }^{3,4}$ Claudine Lacroix, ${ }^{7,8}$ and Sébastien Burdin ${ }^{1,2}$ \\ ${ }^{1}$ Univ. Bordeaux, LOMA, UMR 5798, F-33400 Talence, France \\ ${ }^{2}$ CNRS, LOMA, UMR 5798, F-33400 Talence, France \\ ${ }^{3}$ International Institute of Physics, Universidade Federal do Rio Grande do Norte, 59078-400 Natal-RN, Brazil \\ ${ }^{4}$ Departamento de Física Teórica e Experimental, Universidade Federal do Rio Grande do Norte, 59072-970 Natal-RN,Brazil \\ ${ }^{5}$ Instituto de Física, Universidade Federal do Rio Grande do Sul, 91501-970 Porto Alegre-RS, Brazil \\ ${ }^{6}$ Institut de Physique Théorique, CEA-Saclay, 91191 Gif-sur-Yvette, France \\ ${ }^{7}$ Institut Néel, Université Grenoble-Alpes, F-38042 Grenoble, France \\ ${ }^{8}$ Institut Néel, CNRS, F-38042 Grenoble, France
}

(Received 2 February 2016; published 17 October 2016)

\begin{abstract}
We show how spin-liquid (SL) states can be stabilized in a realistic three-dimensional model as a result of frustration. SU(n)-symmetric generalization of the Heisenberg model for quantum spin $S$ operators is used to investigate the frustrated body-centered tetragonal (BCT) lattice with antiferromagnetic interlayer coupling $J_{1}$ and intralayer first and second-neighbor couplings $J_{2}$ and $J_{3}$. By using complementary representations of the spin operators, we study the phase diagram characterizing the ground state of this system. For small $n$, we find that the most stable solutions correspond to four different families of long-range magnetic orders that are governed by $J_{1}$, $J_{2}$, and $J_{3}$. First, some possible instabilities of these phases are identified for $n=2$, in large $S$ expansions, up to the linear spin-wave corrections. Then, using a fermionic representation of the $\mathrm{SU}(n)$ spin operators for $S=1 / 2$, we find that purely magnetic orders occur for $n \leqslant 3$ while SL solutions are stabilized for $n \geqslant 10$. The SL solution governed by $J_{1}$ breaks the lattice translation symmetry. The modulated SL is associated with a commensurate ordering wave vector $(1,1,1)$. For $4 \leqslant n \leqslant 9$, we show how the competition between $J_{1}, J_{2}$, and $J_{3}$ can turn the magnetically ordered ground state into a SL state. Finally, we discuss the relevance of this scenario for correlated systems with BCT crystal structure.
\end{abstract}

DOI: 10.1103/PhysRevB.94.134420

\section{INTRODUCTION}

In most superconducting materials, magnetic ordering destroys superconductivity. However, magnetic correlations are essential for superconducting pairing in a large variety of unconventional superconductors. In the presence of frustration, classical magnetic order can become unstable against spin-liquid (SL) states, and superconductivity may emerge even with strong magnetic interactions. It is commonly believed that low dimensionality is essential for the formation of a SL, and as result this would also be a crucial ingredient for the realization of superconductors with higher critical temperature. Controverting this idea, we analyze the effect of frustration in the body-centered tetragonal (BCT) lattice, which is one of the 14 existing three-dimensional (3D) lattice structures [1]. This standard crystalline structure is realized in several strongly correlated electron materials with unusual magnetic and transport properties. Among the heavy fermion systems [2,3], different examples of materials with rare-earth atoms on a BCT lattice have been intensively studied for the last few decades: in $\mathrm{URu}_{2} \mathrm{Si}_{2}$, a still-mysterious hidden order (HO) phase appears below the critical temperature $T_{H O} \approx$ $17 \mathrm{~K}$ close to a pressure-induced antiferromagnetic (AF) transition [4,5]; in $\mathrm{YbRh}_{2} \mathrm{Si}_{2}$ and $\mathrm{CeRu}_{2} \mathrm{Si}_{2}$, non-Fermi-liquid properties are observed in the vicinity of the AF quantum phase transitions, which are still poorly understood [6-9]; $\mathrm{CeCu}_{2} \mathrm{Si}_{2}$ was the first heavy-fermion material where unconventional superconductivity was discovered close to an AF transition [10]; $\mathrm{CePd}_{2} \mathrm{Si}_{2}$ also exhibits unconventional superconductivity related to an AF transition [11,12]; multi-Q AF order has been observed in $\mathrm{CeRh}_{2} \mathrm{Si}_{2}$ [13]. It is noticeable that the cuprate superconductors [14] can be included among the AF insulating parent compounds $\mathrm{La}_{2} \mathrm{CuO}_{4}$ and $\mathrm{Sr}_{2} \mathrm{CuO}_{2} \mathrm{Cl}_{2}$ in which the $\mathrm{AF}$ order originates from the $\mathrm{Cu}$ atoms that form a BCT crystal. However the relevant physics in their case is essentially two dimensional (2D) with the BCT structure being involved only in the formation of the square-lattice layers of $\mathrm{Cu}$ atoms that order antiferromagnetically. In this paper we suggest that the rich diversity of unusual physical properties observed in 3D materials with BCT structure is essentially associated with the underlying frustration. To do that we establish direct contact with important theoretical developments which were made in the past few years on the unconventional magnetic properties of the BCT lattice, following the pioneering study by Villain [15-22].

In this article, we analyze the ground states of an $\mathrm{SU}(n)$ generalization of the $J_{1}-J_{2}-J_{3}$ quantum spin $S$ Heisenberg Hamiltonian $H_{n}^{S}$.

\section{CLASSICAL SPIN AND QUANTUM SPIN-WAVE-FLUCTUATIONS APPROACH}

The model Hamiltonian is introduced here, for simplicity, for $n=2$ :

$$
H_{n=2}^{S}=\sum_{\left\langle\mathbf{R}, \mathbf{R}^{\prime}\right\rangle} J_{\mathbf{R} \mathbf{R}^{\prime}} \vec{S}_{\mathbf{R}} \cdot \vec{S}_{\mathbf{R}^{\prime}}
$$

where $\vec{S}_{\mathbf{R}} \equiv\left(S_{\mathbf{R}}^{x}, S_{\mathbf{R}}^{y}, S_{\mathbf{R}}^{z}\right)$ denotes the quantum spin $S$ operators acting on site $\mathbf{R}$ of a BCT-lattice. The antiferromagnetic interaction $J_{\mathbf{R R}^{\prime}}$ connects sites $\mathbf{R}$ and $\mathbf{R}^{\prime}$, and can take three possible values $J_{1}, J_{2}, J_{3}>0$, as indicated in Fig. 1 . 


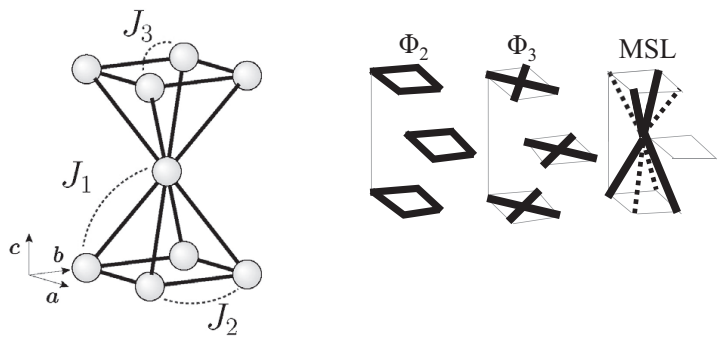

FIG. 1. Left: BCT lattice and the $J_{1}, J_{2}$, and $J_{3}$ interactions. In this work, the tetragonal lattice constants are set to $a=b=c=1$. Right: Bold lines represent the three kinds of intersite SL correlations on the BCT structure.

\section{A. Classical ground state}

Following the standard spin-wave (SW) approach, we start with the $S=+\infty$ generalization of the Hamiltonian (1), which corresponds to the classical spin limit. For the sake of simplification, we consider only magnetic orderings that are characterized by a single wave vector $\mathbf{Q}=2 \pi(\lambda, \mu, \nu)$ identified as $(\lambda, \mu, \nu)$ in reduced notation. Invoking the Fourier transform, this wave vector is used to minimize the classical dispersion,

$$
J(\mathbf{q}) \equiv 8 J_{1} \gamma_{1}^{\mathbf{q}}+2 J_{2} \gamma_{2}^{\mathbf{q}}+4 J_{3} \gamma_{3}^{\mathbf{q}},
$$

with

$$
\begin{gathered}
\gamma_{1}^{\mathbf{q}} \equiv \cos \left(q_{x} / 2\right) \cos \left(q_{y} / 2\right) \cos \left(q_{z} / 2\right), \\
\gamma_{2}^{\mathbf{q}} \equiv \cos \left(q_{x}\right)+\cos \left(q_{y}\right), \\
\gamma_{3}^{\mathbf{q}} \equiv \cos \left(q_{x}\right) \cos \left(q_{y}\right) .
\end{gathered}
$$

By tuning the dimensionless parameters $p_{2} \equiv J_{2} / J_{1}$ and $p_{3} \equiv J_{3} / J_{1}$, we find that the ground state can be characterized by four kinds of possible wave vectors, as depicted in Fig. 2: $\mathbf{Q}_{\mathrm{AF}}^{\mathrm{I}} \equiv(1,1,1)$ and $\mathbf{Q}_{\mathrm{AF}}^{\mathrm{II}} \equiv(1 / 2,1 / 2, v)$ corresponding to the regimes where the Weiss field is dominated by $J_{1}$ and $J_{2}$, respectively. The $v$ degeneracy in the latter case indicates the underlying bidimensionality. The other possible ordering wave vectors are incommensurate and characterize two kinds of helical orders: $\mathbf{Q}_{\text {inc }}^{\text {III }} \equiv\left(0, \Upsilon_{3}, 0\right)$ degenerate with $\left(\Upsilon_{3}, 0,0\right),\left(1, \Upsilon_{3}, 1\right)$, and $\left(\Upsilon_{3}, 1,1\right)$ where $\Upsilon_{3}=\frac{1}{\pi} \arccos \frac{-1}{p_{2}+2 p_{3}}$; and $\mathbf{Q}_{\text {inc }}^{\text {II }} \equiv\left(\Upsilon_{2}, \pm \Upsilon_{2}, 1\right)$ degenerate with $\left(\Upsilon_{2}, 1 \pm \Upsilon_{2}, 0\right)$, where $\Upsilon_{2}=\frac{1}{2 \pi} \arccos \frac{1-p_{2}}{2 p_{3}}$. A different wave vector, $\mathbf{Q}_{\mathrm{AF}}^{\mathrm{III}} \equiv(0,1 / 2, v)$ had been proposed [23] in a $J_{3}$-dominated phase, which corresponds to the commensurate order characterizing a purely bidimensional square lattice. We find that $J_{1} \neq 0$ corrections are relevant and $\mathbf{Q}_{\text {inc }}^{\text {III }}$ is energetically more stable than $\mathbf{Q}_{\mathrm{AF}}^{\mathrm{III}}$. Not surprisingly these two vectors are asymptotically identical at large $p_{3}$. Similarly, $\mathbf{Q}_{\mathrm{inc}}^{\mathrm{II}} \mapsto \mathbf{Q}_{\mathrm{AF}}^{\mathrm{II}}$ at large $p_{2}$. The transition lines separating these four classical phases are given by linear relations between $p_{2}$ and $p_{3}$. The $\mathbf{Q}_{\text {inc }}^{\text {II }}-\mathbf{Q}_{\text {inc }}^{\text {III }}$ transition is discontinuous, the other transitions are continuous.

\section{B. Spin-wave fluctuations}

Next, assuming a given classical ground-state wave vector $\mathbf{Q}$, we investigate the large- $S$ corrections. This expansion

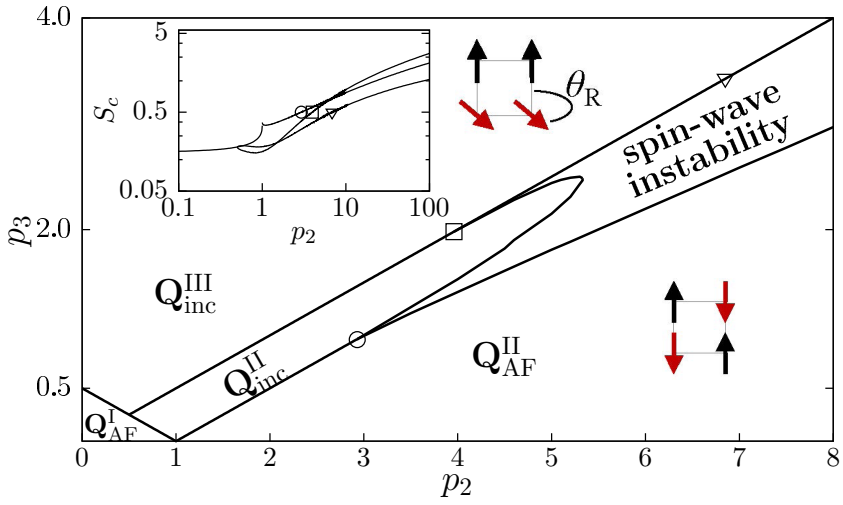

FIG. 2. Classical ground-state phase diagram of the $J_{1}-J_{2}-J_{3}$ model in coordinates $\left(p_{2}, p_{3}\right)$. The solid lines indicate transitions between the stable magnetic ordered states with modulating vectors $\mathbf{Q}_{\mathrm{AF}}^{\mathrm{I}}, \mathbf{Q}_{\mathrm{AF}}^{\mathrm{II}}, \mathbf{Q}_{\mathrm{inc}}^{\mathrm{II}}$, and $\mathbf{Q}_{\mathrm{inc}}^{\mathrm{III}}$. The $\mathrm{SW}$ instability region is computed for $S=1 / 2$. Inset: Critical value $S_{c}$ as a function of $p_{2}$ when $p_{3}$ is bound to the critical lines. The three symbols indicate the corresponding location of critical value $S_{c}=1 / 2$ in each case. Numerical results obtained by using multidimensional Monte Carlo $k$ integration over the BCT Brillouin zone.

invokes a helical generalization of the Holstein-Primakov representation $[16,19,24]$ : introducing boson annihilation (creation) $a_{\mathbf{R}}^{(\dagger)}$ operators, the spin operators are approximated as

$$
S_{\mathbf{R}}^{x} \approx(S / 2)^{\frac{1}{2}}\left(a_{\mathbf{R}}+a_{\mathbf{R}}^{\dagger}\right)
$$

and

$$
\left(\begin{array}{l}
S_{\mathbf{R}}^{z} \\
S_{\mathbf{R}}^{y}
\end{array}\right) \approx\left[\begin{array}{rl}
\cos \theta_{\mathbf{R}} & \sin \theta_{\mathbf{R}} \\
-\sin \theta_{\mathbf{R}} & \cos \theta_{\mathbf{R}}
\end{array}\right]\left(\begin{array}{l}
S-a_{\mathbf{R}}^{\dagger} a_{\mathbf{R}} \\
-i(S / 2)^{\frac{1}{2}}\left(a_{\mathbf{R}}-a_{\mathbf{R}}^{\dagger}\right)
\end{array}\right),
$$

with $\theta_{\mathbf{R}} \equiv \mathbf{Q} \cdot\left(\mathbf{R}-\mathbf{R}_{0}\right)$, where $z$ is the easy axis characterizing a site $\mathbf{R}_{0}$ chosen arbitrarily. The dispersions obtained for the Bogoliubov quasiparticles are

$$
\begin{gathered}
\Omega_{\mathbf{k}}^{+} \equiv J(\mathbf{k})-J(\mathbf{Q}), \\
\Omega_{\mathbf{k}}^{-} \equiv \frac{J(\mathbf{k}+\mathbf{Q})+J(\mathbf{k}-\mathbf{Q})}{2}-J(\mathbf{Q}) .
\end{gathered}
$$

Order by quantum disorder. While the ground-state energy is proportional to $S^{2} J(\mathbf{Q})$ at the highest order, the first correction is proportional to $S \int_{\mathrm{BCT}} d^{3} \mathbf{k} \sqrt{\Omega_{\mathbf{k}}^{+} \Omega_{\mathbf{k}}^{-}}$where the $\mathbf{k}$ integral runs over the first Brillouin zone of the BCT-lattice. Analyzing this correction for the classically degenerate vectors $\mathbf{Q}_{\mathrm{AF}}^{\mathrm{II}}$, we find that the resulting order is stabilized by quantum disorder: the continuous degeneracy is lifted in favor of $v=0$, which is equivalent to $v=1$.

Fluctuation corrections to magnetization. We also studied the effects of fluctuations emerging from the linear SW corrections. Generalizing to the BCT structure the approach introduced in Ref. [25] for the square lattice model, the staggered magnetization is expanded around its classical value $\left\langle S_{\mathbf{R}_{0}}^{z}\right\rangle \approx S-\Delta m\left(p_{2}, p_{3}\right)$. We find

$$
\Delta m\left(p_{2}, p_{3}\right)=\left\langle a_{\mathbf{R}_{\mathbf{0}}}^{\dagger} a_{\mathbf{R}_{\mathbf{0}}}\right\rangle=-\frac{1}{2}+\int_{\mathrm{BCT}} \frac{d^{3} \mathbf{k}}{64 \pi^{3}} \frac{\Omega_{\mathbf{k}}^{+}+\Omega_{\mathbf{k}}^{-}}{\sqrt{\Omega_{\mathbf{k}}^{+} \Omega_{\mathbf{k}}^{-}}} .
$$


Unlike the 2D case [25], the fluctuation corrections do not diverge, which is not surprising for a 3D model. Frustration can relatively increase the critical value of $S$ below which the linear spin-wave correction cancels the staggered magnetization, $S_{c} \equiv \Delta m\left(p_{2}, p_{3}\right)$. Indeed, for a fixed $p_{2}$, we find that $S_{c}$ increases when $p_{3}$ approaches its critical value associated with the classical phase boundary. This maximal value is plotted in the inset of Fig. 2 as a function of $p_{2}$ for the continuous $\mathbf{Q}_{\mathrm{AF}}^{\mathrm{II}} / \mathbf{Q}_{\mathrm{inc}}^{\mathrm{II}}$ transition and on each side of the discontinuous $\mathbf{Q}_{\text {inc }}^{\text {II }} / \mathbf{Q}_{\text {inc }}^{\text {III }}$ transition. On each of these critical lines, we find $S_{c} \sim \sqrt{p_{2}}$ at large $p_{2}$. Furthermore, a logarithmic $S_{c} \sim \ln \left(p_{2} / 2-p_{3}\right)$ and a power law $S_{c} \sim 1 / \sqrt{p_{3}-p_{2} / 2}$ are respectively obtained at large $p_{2}$ in the vicinity of the $\mathbf{Q}_{\text {inc }}^{\text {II }} \rightarrow$ $\mathbf{Q}_{\text {inc }}^{\text {III }}$ and $\mathbf{Q}_{\text {inc }}^{\text {III }} \rightarrow \mathbf{Q}_{\text {inc }}^{\text {II }}$ transitions. This result is consistent with the square lattice spin wave analysis [25].

\section{Discussion}

The SW approach thus reveals some weaknesses of the classical magnetic orders, but the three-dimensionality protects these states against small fluctuations at the lowest, linear order. One may go further and study possible instabilities emerging from the next orders in the $1 / S$ expansion, taking into account interactions between the spin-wave bosonic excitations. However, it has been shown for a two-dimensional lattice that the second-order correction can increase considerably the sublattice magnetization, although the first-order perturbation makes it decrease [26]. It is thus possible that a sign-oscillating series could also characterize the SW expansion from the orders characterizing our model. Therefore, stabilizing a spin-liquid state might require going beyond second order when invoking a perturbative approach from classical order. Hereafter, we bypass this open issue and we follow an alternative and simpler approach.

\section{GENERALIZED SU(n) SYMMETRIC APPROACH}

In this section, we analyze the possibility that the system forms a resonant valence bond state with fermionic excitations and SL correlations $[27,28]$. One of our physical motivations is driven by the physics of unconventional metallic systems with BCT structure: in several of these correlated systems, magnetic degrees of freedom seem to be "deconfined" into fermionic ones that may contribute to the formation of a Fermi surface, unlike weakly coupled bosons. Such a scenario, inspired by the physics of cuprate superconductors [29-32], could be easily strengthened by a coupling of the Heisenberg spins of the $J_{1}-J_{2}-J_{3}$ model to extra charge degrees of freedom. In the following we study this possible "fermionic deconfinement" of spin operators as an intrinsic property of the Heisenberg model. To achieve this goal, considering $S=1 / 2$, the Hamiltonian (1) is generalized to its $\mathrm{SU}(n)$-symmetric form:

$$
H_{n}^{S=1 / 2}=\sum_{\left\langle\mathbf{R}, \mathbf{R}^{\prime}\right\rangle} \frac{J_{\mathbf{R} \mathbf{R}^{\prime}}}{n} \sum_{\sigma \sigma^{\prime}} \chi_{\mathbf{R} \sigma}^{\dagger} \chi_{\mathbf{R} \sigma^{\prime}} \chi_{\mathbf{R}^{\prime} \sigma^{\prime}}^{\dagger} \chi_{\mathbf{R}^{\prime} \sigma},
$$

where $\chi_{\mathbf{R} \sigma}^{(\dagger)}\left(\chi_{\mathbf{R} \sigma}\right)$ are annihilation (creation) fermionic operators with orbital degeneracy $\sigma=1, \ldots, n$ and satisfying the local constraints $\sum_{\sigma} \chi_{\mathbf{R} \sigma}^{\dagger} \chi_{\mathbf{R} \sigma}=n / 2$. This is a $\operatorname{standard} \operatorname{SU}(n)$ generalization [33] of the fermionic representation developed by Abrikosov for $n=2$. The scaling factor $1 / n$ ensures that the energy remains extensive, i.e., proportional to $n$, in the large- $n$ limit.

\section{A. Spin-liquid correlations}

Using the Hubbard-Stratonovich decoupling as described in Refs. [34,35], the low-temperature phases of Hamiltonian (11) can be characterized by two kinds of order parameters: the local magnetization field

$$
m_{\mathbf{R}}^{\sigma}=\left\langle\chi_{\mathbf{R} \sigma}^{\dagger} \chi_{\mathbf{R} \sigma}\right\rangle-\frac{1}{2},
$$

and the intersite spin-liquid fields

$$
\varphi_{\mathbf{R} \mathbf{R}^{\prime}}=-\frac{1}{n} \sum_{\sigma}\left\langle\chi_{\mathbf{R} \sigma}^{\dagger} \chi_{\mathbf{R}^{\prime} \sigma}\right\rangle .
$$

The purely magnetic classical mean-field theory characterized here by a staggered magnetization $m_{\mathbf{R}}^{\sigma}= \pm S_{\mathbf{Q}}$ is equivalent to the one we analyzed for the $S=+\infty$ limit. The corresponding ground-state phase diagram is thus given by Fig. 2. Hereafter, the stability of these classical magnetic orders is analyzed by testing various SL Ansätze as possible alternative ground states. Generalizing the modulated SL (MSL) order introduced elsewhere [35], we consider the nearest-neighbor intersite correlations:

$$
\begin{gathered}
\varphi_{\mathbf{R}^{\prime}}^{1}=\frac{1}{2}\left[\Phi_{1}+i e^{i \mathbf{Q}_{\mathrm{AF}}^{\mathrm{I}} \cdot\left(\frac{\mathbf{R}+\mathbf{R}^{\prime}}{2}\right)} \Phi_{\mathrm{M}}\right], \\
\varphi_{\mathbf{R R}^{\prime}}^{2}=\Phi_{2}, \\
\varphi_{\mathbf{R}^{\prime}}^{3}=\Phi_{3},
\end{gathered}
$$

with a bond index definition similar to that of Fig. 1. The free energy per spin component and per lattice site is expressed as

$$
F=F_{0}-\frac{k_{B} T}{32 \pi^{3}} \int_{\mathrm{BCT}} d^{3} \mathbf{k} \sum_{s= \pm} \ln \left(1+e^{-\frac{E_{\mathbf{k}}^{s}}{k_{B} T}}\right)-\frac{\lambda_{0}}{2},
$$

where $\lambda_{0}$ denotes a Lagrange multiplier that minimizes $F$ in order to satisfy the constraint for the fermionic occupation. For the AF orderings, $F_{0}=-\frac{2 J(\mathbf{Q})}{n}\left|S_{\mathbf{Q}}\right|^{2}$ and the dispersion is $E_{\mathbf{k}}^{ \pm}=\lambda_{0} \pm \frac{J(\mathbf{Q})}{n} S_{\mathbf{Q}}$. For the SL states we find

$$
F_{0} / J_{1}=\left|\Phi_{1}\right|^{2}+\left|\Phi_{M}\right|^{2}+2 p_{2}\left|\Phi_{2}\right|^{2}+2 p_{3}\left|\Phi_{3}\right|^{2}
$$

and

$$
\begin{aligned}
\left(E_{\mathbf{k}}^{ \pm}-\lambda_{0}\right) / J_{1}= & 2 p_{2} \gamma_{2}^{\mathbf{k}} \Phi_{2}+4 p_{3} \gamma_{3}^{\mathbf{k}} \Phi_{3} \\
& \pm 4 \sqrt{\left(\gamma_{1}^{\mathbf{k}} \Phi_{1}\right)^{2}+\left(\gamma_{M}^{\mathbf{k}} \Phi_{M}\right)^{2}}
\end{aligned}
$$

Here, the non-BCT-periodic real term $\Phi_{\mathrm{M}}$ with $\gamma_{\mathrm{M}}^{\mathbf{k}} \equiv$ $\sin \left(q_{x} / 2\right) \sin \left(q_{y} / 2\right) \sin \left(q_{z} / 2\right)$ takes into account a possible spatial amplitude modulation of the SL field. Our numerical analysis is based on self-consistent calculations of the meanfield parameters for each AF or SL state. The most stable solution is then selected by free-energy minimization, invoking Eq. (17). Considering that the MSL is a BCT adaptation of the "kite" phase investigated in Ref. [33] for a square lattice, we also tested another nonhomogeneous "flux" phase SL characterizing a chiral state with complex $\varphi_{\mathbf{R R}^{\prime}}^{1}=\left(\varphi_{\mathbf{R}^{\prime} \mathbf{R}}^{1}\right)^{\star}$ 

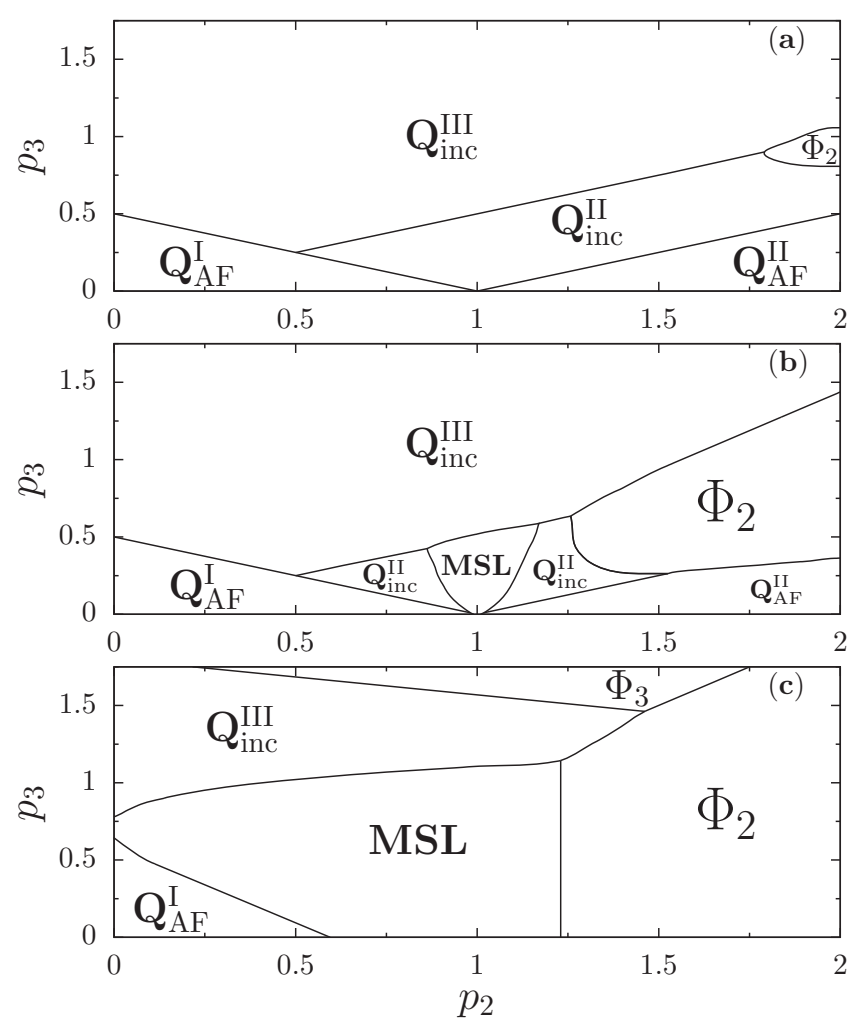

FIG. 3. Ground-state phase diagram of the $J_{1}-J_{2}-J_{3}$ model in coordinates $\left(p_{2}, p_{3}\right)$ computed self-consistently for (a) $n=4$, (b) $n=5$, (c) $n=7$.

that could be described within a very close formalism by simply replacing $\gamma_{\mathrm{M}}^{\mathbf{k}} \rightarrow \sin \left(q_{x} / 2\right) \sin \left(q_{y} / 2\right) \cos \left(q_{z} / 2\right)$. This chiral SL was found to have a higher energy than the MSL.

\section{B. Phase diagram}

For $n \leqslant 3$ we find purely AF ground states, and for $n \geqslant 10$ the most stable states are SL characterized by finite values of either $\Phi_{\mathrm{M}}, \Phi_{2}$, or $\Phi_{3}$. At the mean-field level, these three SL parameters do not coexist. Furthermore, we remark that the transition between the MSL and the $\Phi_{2}$-dominated SL phases is first order. Beyond mean field, we expect that only the SL critical temperature associated with a nonzero $\Phi_{\mathrm{M}}$ still corresponds to a phase transition signaled by the translationsymmetry breaking. For $4 \leqslant n \leqslant 9$ we find a rich phase diagram exhibiting AF and SL quantum phase transitions that are controlled by $J_{1}-J_{2}-J_{3}$ parameters, as illustrated in Fig. 3. Increasing $n$, the AF-SL instability shows up first within the $\mathbf{Q}_{\text {inc }}^{\mathrm{II}}$ phase. Furthermore, comparing the large- $S$ phase diagram (Fig. 2) with that obtained for $n=4$ (Fig. 3), one observes that the $S_{c}=1 / 2$ spin-wave instability is located in the same region where the $\Phi_{2}$-dominant state becomes stabilized. Also, beyond the specificity of the associated order parameters, Fig. 3 indicates that the SL instability "propagates" from large- $p_{2}$ $\left(\Phi_{2}\right)$ to smaller- $p_{2}$ (MSL) areas if we increase the value of $n$.

\section{Modulated spin-liquid phase}

An interesting feature also appears for the MSL solution: with a relatively high numerical accuracy the modulation field $\Phi_{M}$ is found to be always equal to the homogeneous field
$\Phi_{1}$. This leads to a very extreme situation for the interlayer field $\varphi_{\mathbf{R R}^{\prime}}^{1}=\frac{1}{2}\left[\Phi_{1} \pm \Phi_{\mathrm{M}}\right]$ which vanishes on half of the bonds while it keeps the finite value $\Phi_{1}=\Phi_{\mathrm{M}}$ on the other bonds. Introducing the probability $p_{\mathbf{R} \mathbf{R}^{\prime}}^{\text {singlet }}$ that a given bond $\mathbf{R R}^{\prime}$ forms a singlet, the formation of the MSL state can be interpreted here as follows: First, for all the interlayer bonds such that $\mathbf{Q}_{\mathrm{AF}}^{\mathrm{I}} \cdot\left(\mathbf{R}+\mathbf{R}^{\prime}\right) / 2=\pi / 2$, the interaction terms are effectively decoupled at mean-field level, leading to $p_{\mathbf{R R}^{\prime}}^{\text {singlet }}=1 / 4$ and $\left\langle\vec{S}_{\mathbf{R}} \cdot \vec{S}_{\mathbf{R}^{\prime}}\right\rangle=0$. Then the SL with $\left\langle\vec{S}_{\mathbf{R}} \cdot \vec{S}_{\mathbf{R}^{\prime}}\right\rangle \neq 0$ is formed on the other interlayer bonds, with $\mathbf{Q}_{\mathrm{AF}}^{\mathrm{I}} \cdot\left(\mathbf{R}+\mathbf{R}^{\prime}\right) / 2=-\pi / 2$, that remain effectively coupled. Using the numerical value $\Phi_{1}=\Phi_{\mathrm{M}} \approx 0.45$ computed at $T=0$ in the MSL, we find that $p_{\mathbf{R}^{\prime}}^{\text {singlet }} \approx 0.60$ on these effectively coupled bonds. This value has to be compared with the value $\ln (2) \approx 0.69$ that is predicted for a one-dimensional Heisenberg chain by using exact methods [36,37]. We may thus interpret the MSL as a crystal of interacting filaments formed by the connected effectively coupled bonds. In this picture, spin excitations are deconfined fermions moving along these filaments. This may generalize the usual concept of valence-bond crystal [38] where localized spin-1 excitations correspond to confined fermions.

\section{DISCUSSION}

Here we considered a model with only localized spins. However, we know from previous works on heavy fermions and cuprates that charge fluctuations play a crucial role in destabilizing AF states. In the context of the cuprates, the AF phase of the insulating parent compounds corresponds to $\mathbf{Q}_{\mathrm{AF}}^{\mathrm{II}}$. The SL phase introduced by Anderson et al. [28-32] corresponds to the homogeneous correlated state associated here with $\Phi_{2} \neq 0$. One crucial specificity of this SL scenario for superconductivity in cuprates relies on the twodimensionality of the system. Stabilizing a SL state in 3D is commonly thought to be more tricky in view of the fact that the corresponding linear SW correction remains finite within a large- $S$ approach. Nevertheless, we have shown how frustration in the BCT lattice can enhance the critical value $S_{c}$ that, in some sense, characterizes the weakness of AF order against SW fluctuations. In connection with this weakening, we identified various SL phases that can be stabilized when $n$ is larger than a relatively small critical value. The possible emergence of stable SL-like phases in cubic lattices has also been proposed recently by invoking different approximations $[39,40]$. This opens new perspectives for the realization of unconventional electronic quantum orderings in 3D. In particular, our results suggest that the BCT-lattice structure can play a central role in crystalline materials such as the 122 and some cuprates in spite of numerous phases emerging in these systems. Indeed, the very rich phase diagram depicted in Figs. 2 and 3 could provide a unifying framework for understanding and analyzing the intersite correlations in these compounds. The number $n$ may be considered as an effective parameter related to the electronic orbital degeneracy, which could be phenomenologically increased or decreased by charge fluctuations or crystal-field effects. Considering a given compound, $n$ might also be effectively decreased by applying an external magnetic field. Similarly, a tuning of the model parameters $p_{2}$ and $p_{3}$ may phenomenologically account 
for some effects of applied pressure [35]. For example, this scenario could explain two different $\mathrm{AF}$ instabilities of the $\mathrm{HO}$ phase that are observed experimentally in $\mathrm{URu}_{2} \mathrm{Si}_{2}$ : assuming that the HO is a MSL order and applying a finite pressure this corresponds to increasing $J_{1}$ for a fixed $n$ (see Fig. 3), leading to a commensurate AF instability characterized by $\mathbf{Q}_{\mathrm{AF}}^{\mathrm{I}}$, as observed experimentally [4,5]. Alternatively, applying a magnetic field without pressure corresponds to lowering the effective value of $n$ for fixed $p_{2}$ and $p_{3}$ : the MSL (HO) is destabilized to an incommensurate AF. Interestingly, by using different numerical values of $J_{1}, J_{2}$, and $J_{3}$ obtained from different fits of inelastic-neutron-scattering data, our scenario predicts an instability from MSL to $\mathbf{Q}_{\text {inc }}^{\text {III }}=\left(\Upsilon_{3}, 0,0\right)$ with $\Upsilon_{3} \approx 0.69$ (from Ref. [41]), 0.66 (from Ref. [42]), 0.69 (from Ref. [23]), and 0.65 (from Ref. [43]). This scenario could be tested experimentally since it predicts that the AF order $\mathbf{Q}_{\text {inc }}^{\text {III }}$ could be continuously tuned to $\mathbf{Q}_{\mathrm{AF}}^{\mathrm{I}}$ by applying pressure on $\mathrm{URu}_{2} \mathrm{Si}_{2}$ under a high magnetic field.

Invoking the SL instabilities described in this paper we may also generalize to 3D systems the spin-fluctuation pairing mechanism that was proposed in terms of gauge transformations in Refs. [44,45]. Here, the link between the BCT lattice structure and the superconducting order parameter is natural. It can be tested experimentally since we predict that the symmetries of the resulting superconducting order parameters will result from the point-group symmetries of the SL, which may correspond to an in-plane pairing related to $\Phi_{2}$ or $\Phi_{3}$ or a fully 3D pairing associated with $\Phi_{1}$. This SL mechanism driven by frustration on the BCT lattice may also be tested for the heavy-fermion superconductors $\mathrm{CeRu}_{2} \mathrm{Si}_{2}$ and $\mathrm{CePd}_{2} \mathrm{Si}_{2}$, but in these systems valence-fluctuation effects need to be carefully included. The possible formation of a MSL could also give rise to a commensurately ordered pairing that would break the BCT symmetry down to simple tetragonal. Such a modulated pairing unconventional scenario could be tested with the superconducting instability observed in $\mathrm{URu}_{2} \mathrm{Si}_{2}$ inside the HO phase. Alternatively, even if the chiral SL order was found here to be less stable than the MSL, an opposite result could occur by including charge fluctuations.

\section{ACKNOWLEDGMENTS}

We acknowledge the financial support of Capes-Cofecub $\mathrm{Ph}$ 743-12. C.T. is bolsista Capes. This research was also supported in part by the Brazilian Ministry of Science, Technology and Innovation (MCTI) and the Conselho Nacional de Desenvolvimento Científico e Tecnológico (CNPq). Research carried out with the aid of the Computer System of High Performance of the International Institute of Physics-UFRN, Natal, Brazil. The authors are grateful to Frédéric Bourdarot for useful discussions.
[1] C. Kittel, Introduction to Solid State Physics, Eighth Edition (John Wiley \& Sons, New York, 2005).

[2] G. Stewart, Rev. Mod. Phys. 56, 755 (1984).

[3] P. Fulde, P. Thalmeier, and G. Zwicknagl, Solid State Phys. 60, 1 (2006).

[4] T. T. M. Palstra, A. A. Menovsky, J. van deb Berg, A. J. Dirkmaat, P. H. Kes, G. J. Nieuwenhuys, and J. A. Mydosh, Phys. Rev. Lett. 55, 2727 (1985).

[5] J. Mydosh and P. Oppeneer, Rev. Mod. Phys. 83, 1301 (2011).

[6] J. Custers, P. Gegenwart, H. Wilhelm, K. Neumaier, Y. Tokiwa, O. Trovarelli, C. Geibel, F. Steglich, C. Pépin, and P. Coleman, Nature (London) 424, 524 (2003).

[7] S. Friedemann, T. Westerkamp, M. Brando, N. Oeschler, S. Wirth, P. Gegenwart, C. Krellner, C. Geibel, and F. Steglich, Nat. Phys. 5, 465 (2009).

[8] J. Mignot, J. Flouquet, P. Haen, F. Lapierre, L. Puech, and J. Voiron, J. Magn. Magn. Mater. 76-77, 97 (1988).

[9] W. Knafo, S. Raymond, P. Lejay, and J. Flouquet, Nat. Phys. 5, 753 (2009).

[10] F. Steglich, J. Aarts, C. D. Bredl, W. Lieke, D. Meschede, W. Franz, and H. Schäfer, Phys. Rev. Lett. 43, 1892 (1979).

[11] N. Mathur, F. Grosche, S. Julian, I. Walker, D. Freye, R. Haselwimmer, and G. Lonzarich, Nature (London) 394, 39 (1998).

[12] A. Demuer, D. Jaccard, I. Sheikin, S. Raymond, B. Salce, J. Thomasson, D. Braithwaite, and J. Flouquet, J. Phys.: Condens. Matter 13, 9335 (2001).

[13] S. Kawarazaki, M. Sato, Y. Miyako, N. Chigusa, K. Watanabe, N. Metoki, Y. Koike, and M. Nishi, Phys. Rev. B 61, 4167 (2000).
[14] J. Bednorz and K. Muller, Z. Phys. B: Condens. Matter 64, 189 (1986).

[15] J. Villain, J. Phys. Chem. Solids 11, 303 (1959).

[16] H. Diep, Phys. Rev. B 40, 741 (1989).

[17] H. Diep, Phys. Rev. B 39, 397 (1989).

[18] E. Rastelli, S. Sedazzari, and A. Tassi, J. Phys.: Condens. Matter 1, 4735 (1989).

[19] E. Rastelli, S. Sedazzari, and A. Tassi, J. Phys.: Condens. Matter 2, 8935 (1990).

[20] R. Quartu and H. Diep, J. Magn. Magn. Mater. 182, 38 (1998).

[21] D. Loison, Phys. A (Amsterdam, Neth.) 275, 207 (2000).

[22] A. Sorokin and A. Syromyatnikov, J. Exp. Theor. Phys. 113, 673 (2011).

[23] K. Sugiyama, H. Fuke, K. Kindo, K. Shimoata, A. Menovsky, J. Mydosh, and M. Date, J. Phys. Soc. Jpn. 59, 3331 (1990).

[24] A. Chubukov, J. Phys. C: Solid State Phys. 17, L991 (1984).

[25] P. Chandra and B. Douçot, Phys. Rev. B 38, 9335(R) (1988).

[26] J.-i. Igarashi and T. Nagao, Phys. Rev. B 72, 014403 (2005).

[27] A. Aharony and B. Huberman, J. Phys. C: Solid State Phys. 9, L465 (1976).

[28] P. Fazekas and P. Anderson, Philos. Mag. (1798-1977) 30, 423 (1974).

[29] P. W. Anderson, G. Baskaran, Z. Zou, and T. Hsu, Phys. Rev. Lett. 58, 2790 (1987).

[30] G. Baskaran, Z. Zou, and P. Anderson, Solid State Commun. 63, 973 (1987).

[31] T. Rice, S. Gopalan, and M. Sigrist, Europhys. Lett. 23, 445 (1993).

[32] X.-G. Wen and P. A. Lee, Phys. Rev. Lett. 76, 503 (1996). 
[33] I. Affleck and J. D. Marston, Phys. Rev. B 37, 3774(R) (1988).

[34] C. Pépin, M. R. Norman, S. Burdin, and A. Ferraz, Phys. Rev. Lett. 106, 106601 (2011).

[35] C. Thomas, S. Burdin, C. Pépin, and A. Ferraz, Phys. Rev. B 87, 014422 (2013).

[36] H. Bethe, Eur. Phys. J. A 71, 205 (1931).

[37] U. Schollwock, Rev. Mod. Phys. 77, 259 (2005).

[38] Introduction to Frustrated Magnetism, Series in Solid-State Sciences, edited by C. Lacroix, P. Mendels, and F. Mila (Springer, New York, 2011), Vol. 164.

[39] M. Laubach, D. G. Joshi, J. Reuther, R. Thomale, M. Vojta, and S. Rachel, Phys. Rev. B 93, 041106(R) (2016).
[40] D. J. J. Farnell, O. Götze, and J. Richter, Phys. Rev. B 93, 235123 (2016).

[41] C. Broholm, H. Lin, P. T. Matthews, T. E. Mason, W. J. L. Buyers, M. F. Collins, A. A. Menovsky, J. A. Mydosh, and J. K. Kjems, Phys. Rev. B 43, 12809 (1991).

[42] H. Kusunose, J. Phys. Soc. Jpn. 81, 023704 (2012).

[43] F. Bourdarot, Habilitation thesis, Grenoble, 2013 (unpublished).

[44] P. A. Lee, N. Nagaosa, and X. G. Wen, Rev. Mod. Phys. 78, 17 (2006).

[45] X.-G. Wen, Quantum Field Theory of Many-Body Systems: From the Origin of Sound to an Origin of Light and Electrons, Oxford Graduate Texts (Oxford University Press, Oxford, 2004). 